\title{
Scientific and regulatory approaches to confirm quality and improve patient perceptions of generic drug products in Japan
}

\author{
Hiroko Shibata', Hiroyuki Yoshida ${ }^{1}$, Ken-ichi Izutsu ${ }^{1 *}$ (D), Chikako Yomota ${ }^{1,2}$, Yukihiro Goda ${ }^{1}$ and Haruhiro Okuda ${ }^{1}$
}

\begin{abstract}
The rapidly growing medical expense going with aging population in Japan requires the use of generic products derived from off-patent active pharmaceutical ingredients (APIs) formulations to reduce the financial burden for the national health insurance system, while at the same time avoiding undermining the quality of medical care. This article provides an overview of the regulatory approaches available to confirm the quality of generic products and gain their greater acceptance by patients. Among several approaches taken by the Ministry of Health, Labor, and Welfare (MHLW), designing systems to supply higher quality products, and providing scientific information to patients and healthcare professionals are key elements to promote the voluntary choice of the generic product. The revision of bioequivalence guidelines and the enhancement of good manufacturing practice (GMP) requirements facilitate the rational development and manufacturing control of generic formulations. A program termed Quality Reevaluation of Ethical Drugs was carried out from 1997 to 2012 using dissolution tests to avoid any significant biolNequivalence between originators and generic oral formulations. The evaluation of product quality and the assessment of the literature information by the Expert Committee on Quality of Generic Drug Products have provided a unique science-based and patient-focused approach for the distribution of reliable generic products. Some current and future issues regarding complex generic drugs are also discussed.
\end{abstract}

Keywords: Quality, Generic drugs, Japan, Expert Committee on Quality of Generic Drug Products, Bioequivalence

\section{Background}

The rapidly aging population, which is growing at the fastest speed among countries belonging to the Organization for Economic Cooperation and Development (OECD), makes the increasing gross medical care expenditure a major social issue in Japan (OECD 2015). The Ministry of Health, Labor, and Welfare (MHLW) is promoting the use of generic drugs, introduced after the patent has expired and the original drug reexamination period (usually 8 years) has passed, instead of off-patented brand products, in order to reduce the financial burden for patients and the health insurance system (MHLW 2012b). Regulations supporting development and continuous supply of

\footnotetext{
* Correspondence: izutsu@nihs.go.jp

'Drug Division, National Institute of Health Sciences, 1-18-1 Kamiyoga,

Setagaya-ku, Tokyo 158-8501, Japan

Full list of author information is available at the end of the article
}

generic products therapeutically equivalent to the innovator formulations is a major challenges in many countries. Guidelines for bioequivalence (BE) studies and GMP regulations applied for the development and manufacturing of generic products are similar to those in other countries, while the requirements [e.g., absence of biopharmaceutics classification system (BCS) biowaiver for new generic products] have some differences (Davit et al. 2013; MHLW 2012a). In addition, some unique measures to relieve concerns over the quality of generic drug products by healthcare professionals and patients and to assist with their choice need to be taken. This article provides an overview of the regulatory scientific approaches to distribute reliable generic pharmaceuticals and to increase their acceptance in Japan. Some background information has been provided in addition to the description of quality-related challenges regarding complex generic 
drugs. Follow-on biologics and crude drugs are beyond the focus of this review. The opinions expressed in this article are the author's own and do not reflect the view of the organization.

\section{General background}

A generic drug is defined as a drug that is interchangeable with the original reference drug, contains the same amount of the same active pharmaceutical ingredient (API), and has the same administration routes, indication, therapeutic effects, and dosage regimen as the original drug. A decade ago, the use of generic drugs in Japan was much more limited (18.7\% of volume in 2007) than those in US and EU countries (over $50 \%$ ).

There were three major reasons that prevented an increasing usage of generic drugs in Japan. (a) Several surveys indicated that one of the major reasons for not choosing generic products was concerns over product quality (Fujimura and Watanabe 2012). Moreover, some reports on substandard generic drugs in other countries also raised anxiety (Vial et al. 2008). (b) Relatively small economic incentive to use generic drugs under universal health insurance systems were considered another reason for their discrete use (Takizawa et al. 2015). Indeed, both the National Health Insurance and the Employees' Health Insurance systems set relatively high official drug prices for generic ethical pharmaceuticals $(60-70 \%$ until 2013 , currently $50-60 \%$ of the corresponding brand product), and low copayment ratio (usually below $30 \%$ for many insured clients), which reduces difference in each patient's payment and their choice of generic products. (c) The general patient's preference for branded products and the absence of formulation substitution rights for pharmacists resulted in a barrier that prevents usage of generic drug products. The aging society and the relatively high official price for generic products promoted the development of many "easy-to-swallow" oral formulations implementations, such as changes from capsules to tablets, and tablets downsizing.

The MHLW set two different programs in 2007 and 2013, respectively, entitled "Action Program for the Promotion of the Safe Use of Generic Drugs" (OECD 2009) and "Roadmap for further use of generic drugs" (MHLW 2012b). These programs consist of measures for stable supply, assurance of quality, provision of medical and product information from manufacturers, improvement of the environment for the promotion of use of generic drugs, and matters concerning the medical insurance system.

\section{Review and approval of generic drugs in Japan}

The Pharmaceuticals and Medical Devices Agency (PMDA) reviews the equivalence between generic and originator products from the perspective of quality, efficacy, and safety based on documents submitted by the applicants (Kuribayashi et al. 2015). The application for new generic drugs includes manufacturing methods, specifications and test methods, stability test data performed in accelerated conditions, as well as BE tests. The PMDA requires the generic products to be "comparable or better" that the originator product concerning containers and storage requirements, period of validity, specification, and quality control test methods (PMDA 2014). The new generic drugs are approved by the MHLW after GMP inspection. Review of generic products applications based on Common Technical Document (CTD) format is now under preparation by the PMDA and industry bodies.

\section{Official testing and production control by GMP}

The MHLW together with the National Institute of Health Sciences (NIHS), the National Institute of Infectious Diseases (NIID), and the prefectural health institutes, runs official testing of the pharmaceutical products available on the market, as a part of the Simultaneous Monitoring \& Guidance Program. The tests mainly focusing on assay, purity, and dissolution specifications are conducted every year for 20-30 APIs (300-900 pharmaceutical products). A few non-conforming products ( $0.3 \%$ in the fiscal years (FY) 2008-2014) may be recalled based on the Pharmaceutical and Medical Device Act. Reports of adulterated or counterfeit ethical pharmaceuticals are very rare in Japan, since most of the ethical pharmaceutical products are distributed through authorized suppliers or directly by manufacturers. There are, however, multiple cases of fake or substandard products purchased through overseas Internet pharmacy sites for personal use (Baert and De Spiegeleer 2010; Khan et al. 2012).

Various guidelines harmonized in the International Conference on Harmonization of Technical Requirements for Registration of Pharmaceuticals for Human Use (ICH) (e.g., Good Manufacturing Practice guide for Active Pharmaceutical Ingredients) and originally prepared for new drug products, also play important roles in the production of high quality generic drug products. Their details, however, are above the focus of this review. Japan joined the Pharmaceutical Inspection Convention and Pharmaceutical Inspection Co-operation scheme (PIC/S), an international body promoting GMP standards, in July 2014. The NIHS, NIID, and prefectural health institutes have been certified by the GMP inspectorate as Official Medicines Control Laboratories (OMCL) owing to their high analytical skills and a quality system based on ISO 17025. About $60 \%$ of APIs or API intermediates used for generic drug products are imported (MUFJRC 2014). Operating global GMP standards will improve the quality of drugs manufactured in Japan and promote their export competitiveness (Katori 2014). 


\section{Bioequivalence guidelines}

The current set of guidelines for BE studies is listed in Table 1 (MHLW 2012a). The "Guideline for BE studies for generic products" serves as a core guideline. The content of another guideline entitled: "Guideline for bioequivalence studies for formulation changes of oral solid dosage forms" corresponds to the FDA SUPAC-IR and -MR (scale-up and post-approval changes, immediate release, and modified release solid oral dosage form). Other guidelines describe requirements in the development of different strengths or different dosage for oral solid formulations. Despite the fact that the basic concept underlying the Japanese $\mathrm{BE}$ guidelines is identical to that of the FDA, EMA, and WHO, there are some differences.

The most apparent difference is the absence of a biopharmaceutics classification system (BCS)-based biowaiver in the application of new generic products. Two major reasons for not adopting a BCS-based biowaiver system at the previous revisions were (1) the difficulty in measuring accurate and reliable value of membrane permeation for each API, and (2) the insufficient available data to determine whether the system appropriately reveals the lack of bioequivalence (bioINequivalence) caused by the formulation and manufacturing processes.

Another difference in the guidelines was stipulated to mitigate bioavailability-related risks in patients with lower stomach acid. The ratio of achlorhydric (i.e., hypochlorhydric) patients in Japan is higher compared to that in US and EU countries, particularly among the elderly population (Morihara et al. 2001). Thus, the guidelines requires $\mathrm{BE}$ tests to be performed on subjects with low gastric acidity, if the test formulation shows a dissolution profile different from the reference product in neutral $(\mathrm{pH}$ 6.8) media. For drugs used only for a specific group of patients, the guidelines recommend $\mathrm{BE}$ tests to be performed with the specific population, if a large difference in dissolution profiles is present.

For oral extended-release products, the guideline requires the new generic products to have a dissolution

Table 1 Current set of major guidelines for bioequivalence studies in Japan (published from MHLW)

Guideline for Bioequivalence Studies of Generic Products

Guideline for Bioequivalence Studies for Formulation Changes of Oral Solid Dosage Forms

Guideline for Bioequivalence Studies of Generic Products for Different Strengths of Oral Solid Dosage Forms

Guideline for bioequivalence studies for different oral solid dosage forms

Bioequivalence Studies of Generic Products for Ethical Combination Drug Products

Bioequivalence Studies for Different Strengths of Ethical Combination Drug Products and Formulation Changes of Ethical Combination Drug Products

Guidance for Bioequivalence Studies of Generic Products for Topical Use profile similar to that of the reference products in multiple test conditions (e.g., methods, medias, and rotation speeds) before undertaking a human BE study. It also recommends that the test product should not differ noticeably from the reference product in size, shape, or specific gravity. This also applies to the $\mathrm{BE}$ test for products with different strengths and for formulation changes. The current Japanese guideline does not adopt in vitro and in vivo correlation for oral extended-release formulations.

The guidelines require human $\mathrm{BE}$ studies in healthy subjects for the development of all new generic products except for certain high-risk drugs studied by groups of patients. The bioavailability of orally administered drug products are compared using the area under the curve (AUC) and maximum concentration $\left(C_{\max }\right)$ as key parameters.

Implementation of the BCS-based biowaiver system in this decade has provided information in support to the approaches used in the US and EU, inducing its adoption in many other countries (International Pharmaceutical Federation (FIP); García-Arieta and Gordon 2012; Uppoor et al. 2014; WHO 2011). At present, the MHLW and PMDA are reevaluating the clinical significance of the BCS-based biowaiver and its consistency with current guidelines and regulations. Adopting the BCS-based biowaiver system would reduce the regulatory burden in the development of new generic drugs and mitigate potential risks for healthy volunteers participating in clinical studies.

The Japanese BE guideline for topical dermatological drugs mainly requires in vivo dermatopharmacokinetic studies based on measurements of drug absorption into the stratum corneum. The guideline also allows other methodologies, including BE studies with pharmacodynamic (PD) and pharmacokinetic (PK) endpoints, and measurements of the unabsorbed drug. The appropriate choice of the evaluation method to use, including new techniques (to meet the nature of API, drug formulation, and target skin layer) should help in providing better therapeutic equivalence (Braddy et al. 2015). How to prove the equivalence of some topical generic products developed without human BE studies before the current guideline were in place, is still an issue for the supply of reliable generic drugs.

\section{Quality reevaluation of ethical drugs program}

Dissolution tests played an important role in the "Quality Reevaluation of Ethical Drugs (QRED)" program ran by the MHLW from 1997 to 2012, with the purpose of improving and maintaining the quality of oral generic drug products (Yomota 2012). Dissolution testing was included as a required specification for both originator and generic drug products in 1995 in order to improve their performance consistency between batches. 
While most of the generic drug products after 1980 were developed with human $\mathrm{BE}$ studies, the absence of dissolution specification in both the originator and corresponding generic products prior to 1995, suggested a lack of performance control. The possible large difference in bioavailability between originator and generic formulations developed without dissolution specifications due to relatively poor methods to control manufacturing procedures at the time became a great concern. The core activity of the QRED program was to perform dissolution tests on originator drugs approved before March 1995 by the NIHS and 10 prefectural health institutes, using several test media, and to match their generic products in terms of dissolution profile through formulation and process changes. Unified dissolution specifications for each formulation were also set in the program. The reevaluation results of 638 APIs (4,588 products) performed until 2012 were reported, and the specifications and profiles of dissolution tests were published as the Japanese Orange Books. Technical difficulties (e.g., analysis, large variation between batches) in a limited number of formulations hindered setting the dissolution specification in this program.

The purpose of the above measure was to indirectly ensure $\mathrm{BE}$ between the original and generic products by coordinating dissolution profiles. The efforts were based on the concept that the risk of significant difference in bioavailability should be minimized if the dissolution profiles of the originator and generic product are similar under several test conditions. It was not realistic, although desirable, to conduct new human studies on several products in a short period. New BE studies were required for products that needed extensive changes in the formulation composition to achieve a dissolution profile similar to that of the reference product. The manufacturers are now recommended to confirm the dissolution profiles on a regular basis following the program implementations.

\section{Activities of the expert committee on quality of generic drug products}

The background analysis performed before implementation of the MHLW program to promote the use of generic pharmaceuticals, indicated the necessity for active measures to improve their recognition by healthcare professionals and patients, rather than just showing compliance with product specifications and GMP. Thus, in 2008, the NIHS organized the Expert Committee on Quality of Generic Drug Products under the cooperation of MHLW and PMDA. The Committee, consisting of 12-14 experts from medical professions and academia, has deliberated over a wide range of quality-related issues (Table 2). Moreover, by evaluating the relevance of the reports about the quality problems of generic products, the Committee has
Table 2 Expert Committee on Quality of Generic Drug Products

\begin{tabular}{|c|c|}
\hline Organizer & $\begin{array}{l}\text { Ministry of Health, Labor, and Welfare (MHLW) } \\
\text { National Institute of Health Sciences (NIHS) } \\
\text { Pharmaceutical and Medical Devices Agency } \\
\text { (PMDA) }\end{array}$ \\
\hline Members (12-14) & $\begin{array}{l}\text { Academia } \\
\text { Representatives from Japan Medical Association, } \\
\text { Japan Dental Association, Japan Pharmaceutical } \\
\text { Association }\end{array}$ \\
\hline $\begin{array}{l}\text { Observer Industry } \\
\text { Associations }\end{array}$ & $\begin{array}{l}\text { Japan Generic Medicines Association } \\
\text { Federation of Pharmaceutical Manufacturers } \\
\text { AssociationsLiterature of Japan }\end{array}$ \\
\hline $\begin{array}{l}\text { Information } \\
\text { Evaluations }\end{array}$ & $\begin{array}{l}\text { Scientific reports relating to quality or clinical } \\
\text { efficacy/safety of generic products Content of } \\
\text { concultations received by a consultatin service } \\
\text { counter in PMDA }\end{array}$ \\
\hline Tests and Evaluations & Purity, dissolution, others \\
\hline $\begin{array}{l}\text { Working Group on } \\
\text { Formulation Testing }\end{array}$ & $\begin{array}{l}\text { NIHS and } 10 \text { Prefectural Institutes of Health } \\
\text { (Aichi, Fukuoka, Hyogo, Kanagawa, Kyoto, Osaka, } \\
\text { Saitama, Shizuoka, Toyama, Tokyo) }\end{array}$ \\
\hline $\begin{array}{l}\text { Specific working } \\
\text { group }\end{array}$ & $\begin{array}{l}\text { Itraconazole capsule WG, Spherical adsorbent } \\
\text { carbon WG }\end{array}$ \\
\hline
\end{tabular}

recommended the necessary actions that needed to be implemented by regulatory bodies (e.g., MHLW, PMDA) and by the industry. Some of the Committee requests were outside the legal requirements set under the Pharmaceutical and Medical Device Act. The Committee has evaluated the relevance of information regarding quality, safety, and efficacy topics reported in scientific journals, or at conference presentations. The contents of the quality-related inquiries issued at the Consultation Service Counter of the PMDA as well as the agenda proposed by the members have been also discussed. A Formulation Evaluation Working Group (hereafter called Formulation WG), composed of NIHS and 10 prefectural health institutes, has conducted tests and analyses for the confirmation of problems reported. The combination of deliberation and testing/evaluation makesthe Committee unique in its patient-focused approach for the distribution of generic pharmaceuticals.

With the increased use of generic drugs, the numbers of reports concerning the quality, efficacy, or safety problems of some products increased in the journals and the meetings for some medical and pharmaceutical societies. The content of 60-80 research articles and conference presentations, regarding the quality and/or the clinical effects of generic drug products in Japan, has been assessed by the committee members every year to determine their relevance. The Japan Generic Medicines Association (JGA) and The Federation of Pharmaceutical Manufacturers' Association of Japan (FPMAJ) have assisted the Committee by listing the presentations, additional information provided by authors of the articles, and actions, or comments delivered by the corresponding manufacturers. The Committee has recommended some government actions or further investigations by divisions 
in the MHLW and PMDA concerning safety issues. Some non-critical issues relating to patient preferences (e.g., flavors, textures) or applicability of the formulations in ways outside of the recommended procedures have not been the subjects of the Committee. The gathering of information, however, has provided the manufacturers with a chance for product improvement. For example, bitterness of granules used in pediatric syrup and orodispersible tablets, or the usability of ophthalmic solutions could affect patients' adherence. The Committee has published all the data used in the semi-annual plenary meeting online to keep the transparency of the activities and to provide information for patients, healthcare professionals, and manufacturers (ECQGDP 2016).

\section{Formulation working group of the committee}

The examinations or tests of objects have been conducted by the Formulation WG. The WG, unlike the Simultaneous Monitoring \& Guidance, has also studied items that are usually not set in the specification. Such tests/items include impurity analysis and comparison of in vitro dissolution profiles using multiple test media in order to assure that the quality of the generic drugs will be equivalent to or more than the original drugs. These tests have been conducted for 82 formulations (1163 products) by the Formulation WG (Table 3) (ECQGDP 2016). Specific working groups were organized for more complex issues (e.g. itraconazole capsules and spherical adsorbent carbon formulations), as described below.

\section{Purity of injections}

The background analysis also showed the preference of many healthcare professionals for branded products particularly when it comes to injection formulations. There was a widespread but groundless belief that many generic drugs contain higher impurities, while meeting specifications. Thus, the Committee decided to have the Formulation WG perform analysis of the injection formulations, and presented the results. Until 2014, the WG analyzed the purity and composition of related substances present in 134 products (14 APIs) (Table 4). The formulations containing certain APIs with relatively high risks and benefits were chosen for the analysis.

Table 3 Testing types and number of formulations or products tested by the Formulation WG

\begin{tabular}{lll}
\hline Test & Formulations & Products \\
\hline Dissolution & 64 & 952 \\
Purity & 13 & 113 \\
Assay & 2 & 27 \\
Uniformity of dosage units & 2 & 58 \\
Composition & 1 & 13 \\
Total & 82 & 1163 \\
\hline
\end{tabular}

Cisplatin is a typical API that requires controlled administration with clinical monitoring, due to the possible nephrotoxic effects and other risks. HPLC analysis through several methods (listed in the Japanese Pharmacopoeia (JP), European Pharmacopoeia (EP), and in the specification tests) showed no apparent differences in purity level between the originator and generic products, at least in the ones tested. Injection formulations of Teicoplanin (a fermentative antibiotic consisting of six principal components), showed composition ratios within the limits listed in the JP and EP.

Analysis of many other injectable generic products showed that they not only met the purity specification, but also contained similar compositions of related substances to those in the original products except in the case of two APIs. One of them includes the observation of some impurities found in two generic ritodrine hydrochloride formulations (used to stop premature labor), but not in the originator product. The Committee recommended the manufacturer for a root-cause analysis and improvement of the formulations. The manufacturer found that the impurity was a sulfite ion adduct potentially formed during the heat sterilization process. After implementation of the process improvements and changes in the API suppliers, the Formulation WG confirmed the decrease of impurities in these generic products to a level comparable to those in the original product. Some reports suggested higher proportion of adverse effects in some generic ritodrine products, although the relationship between their quality and clinical observation was not clear (Umezawa and Fujita 2013). The other case involved the presence of a small amount of phenol in two elcatonin injection formulations that was described as process-related impurity.

\section{Dissolution profiles}

The Committee set higher standards for dissolution of oral generic drug formulations than those in place for their specifications by comparing the dissolution profiles of the generic with those of originator products. The Formulation WG performed dissolution tests of originator and generic products in the four test media (water and three buffers), taking dissolution profiles at multiple time points. Similarity of the dissolution profiles between the originator and generic formulations were examined by modified F2 criteria (with specific range for F2 values) or by differences in the average dissolution ratio at particular time points. The approach is based on the concept described in the BE guidelines and QRED program. The purpose of this evaluation was to confirm consistency of the dissolution profiles with those of the original product found at the time of approval.

Similarities of the dissolution profiles with the corresponding originator product obtained during the QRED 
Table 4 Number of injections tested for purity by the Formulation WG

\begin{tabular}{|c|c|c|c|c|}
\hline API & Formulation & Strength & Products & Method \\
\hline Cefazolin sodium & $\begin{array}{l}\text { Injection/sterile powders } \\
\text { for injection }\end{array}$ & $1 \mathrm{~g} / \mathrm{vial}$ & 5 & JP (formulation) \\
\hline Cisplatin & Injection/solutions & 10 mg/vial & 6 & $\begin{array}{l}\text { JP (API), EP, Approval } \\
\text { documentation }\end{array}$ \\
\hline Elcatonin injection & Injection/solutions & 10 elcatonin/1 mL/vial & 13 & $J P(A P I)$ \\
\hline \multirow[t]{2}{*}{ Famotidine } & $\begin{array}{l}\text { Injection/sterile powders } \\
\text { for injection }\end{array}$ & 20 mg/vial & 6 & JP (formulation) \\
\hline & Injection/solutions & 20 mg/vial & 1 & Approval documentation \\
\hline lopamidol & Injection/solutions & $61.24 \% 50$ mL/vial & 4 & $J P(A P I)$ \\
\hline $\begin{array}{l}\text { Minocycline hydrochloride } \\
\text { for injection }\end{array}$ & $\begin{array}{l}\text { Injection/sterile powders } \\
\text { for injection }\end{array}$ & 100 mg/vial & 6 & JP (formulation) \\
\hline Nafamostat mesilate & $\begin{array}{l}\text { Injection/sterile powders } \\
\text { for injection }\end{array}$ & 10 mg/vial & 15 & Approval documentation \\
\hline Nicardipine hydrochloride & Injection/solutions & 10 mg/vial & 7 & JP (formulation) \\
\hline \multirow[t]{2}{*}{ Ozagrel sodium } & $\begin{array}{l}\text { Injection/sterile powders } \\
\text { for injection }\end{array}$ & $20 \mathrm{mg} / \mathrm{vial}$ & 11 & JP (formulation) \\
\hline & Injection/solutions & 20 mg/vial, 80 mg/vial & 5 & Approval documentation \\
\hline Piperacillin sodium & $\begin{array}{l}\text { Injection/sterile powders } \\
\text { for injection }\end{array}$ & $1 \mathrm{~g} / \mathrm{vial}$ & 7 & JP (formulation) \\
\hline Ritodrine hydrochloride & Injection/solutions & $50 \mathrm{mg} / \mathrm{vial}$ & 10 & $J P(A P I)$ \\
\hline $\begin{array}{l}\text { Sulbactam sodium/Ampicillin } \\
\text { sodium }\end{array}$ & $\begin{array}{l}\text { Injection/sterile powders } \\
\text { for injection }\end{array}$ & $1.5 \mathrm{~g} / \mathrm{vial}$ & 9 & Approval documentation \\
\hline Vancomycin hydrochloride & $\begin{array}{l}\text { Injection/sterile powders } \\
\text { for injection }\end{array}$ & $0.5 \mathrm{~g} / \mathrm{vial}$ & 8 & JP (formulation) \\
\hline Total & 13 formulations & & 113 products & \\
\hline
\end{tabular}

program were also examined for formulations listed in the Japanese Orange Book. Those showing dissolution profiles matching at least one of the two sets of control dissolution profiles were defined as "formulations showing similar dissolutions". The use of two profiles is to account for possible changes occurring in the originator product profiles. Some generic formulations developed after the QRED program confirmed BE with the originator product in the different dissolution profiles. Thus, the Committee is now discussing ways to confirm consistency of the dissolution behavior within the development batch (i.e., biobatch). Confirming dissolution behavior similarities and/or consistencies in multiple media is considered a rational way to prevent large differences in the bioavailability during the product lifecycle, although this is not a legal requirement.

The Formulation WG has conducted dissolution tests for 70 formulations (952 products) until September 2015. These include formulations selected from the therapeutic category of drugs classified a s antihypertensive, hypnotics and sedatives, anxiolytics, antidiabetics, as well as drugs considered important from the literature survey. The generic products found outside the range of similarities were approximately $4 \%$ of all tested products. The Committee recommended the corresponding manufacturers to better control the product performance. After the investigation and formulation improvement, the similarity in dissolution behaviors was reconfirmed in each product by the Formulation WG. Manufacturers' analysis suggested several factors that might have induced changes in the dissolution behavior. They include issues related to the materials used (e.g., crystal size of API and moisture content of excipients) or to the manufacturing process (e.g., coating condition). The only product that did not meet its dissolution specification was recalled. Dissolution profile of the particular product also failed to meet the similarity range.

The process to evaluate the dissolution behavior of generic products also revealed some quality-related issues in off-patented originator products. An originator diazepam product, which marketing authorization was transferred from another company, showed changes in the dissolution behaviors from those obtained in the QRED program. Reviewing the granulation and compression process resulted in the dissolution profiles close to those in the QRED program. Another company improved manufacturing control of bezafibrate SR tablet product to reduce variation in the dissolution profiles within batches, after the Committee found the dissolution was close to its specification. An itraconazole capsule product showed large variation in dissolution (see below). These findings 
confirmed the importance of the product quality control throughout the product lifecycle, including technology transfer after patent expiration.

\section{Itraconazole capsules}

Itraconazole capsules are mainly used as antifungals to treat superficial or deep mycosis. The originator itraconazole capsule uses polymer-based solid dispersion to improve dissolution and gastric absorption, since the crystal shows a typical low solubility (Janssens and Van den Mooter 2009). There were some reports suggesting differences in bioavailability between originator and the generic products (Toyoguchi et al. 2005). The working group organized in the Generic Committee focusing on itraconazole found batch-to-batch variations in dissolution profiles of the originator capsule product, which suggested possible differences in the performance of reference batches for the development of generic products. Thus, the Committee requested the innovator company to run a human $\mathrm{BE}$ study using different commercially available batches of capsule formulations that showed fast and slow dissolution rates. The study was performed in 2010 and showed elevated $\mathrm{C}_{\max }$ and AUC in the high dissolution capsule formulation. Neither $C_{\max }$ nor AUC showed the test batch to be bioequivalent to the reference batch, which was reported at the 5th meeting of the Committee (Fig. 1, Table 5) (ECQGDP 2010). The batch records showed an increasing formulation dissolution rate starting from 2006 with some variability, despite all of them meeting product specification. The Committee recommended the innovator company to improve the process in order to minimize the batch-to-batch variation in dissolution profile and to meet the target bioavailability, after confirming the absence of possible adverse effect cases in clinical reports. The case indicated the relevance of process control and appropriate characterization methods application, to keep the dissolution and bioavailability of low solubility APIs categorized (BCS 2 or 4), particularly in formulations that use dissolution-enhancing technologies.

\section{Spherical adsorbent carbon}

Oral spherical adsorbent carbon, originally approved in Japan in 1991, is used for patients with chronic renal failure to prolong the time to initiation of kidney dialysis, by adsorbing and eliminating uremic toxins in the gastrointestinal tract (Ueda et al. 2007). Some generic preparations have been approved because of pharmacological effects (e.g., decrease of serum uremic toxin in rats) similarities with those of the original product, according to the $\mathrm{BE}$ guideline available at the time. Any existing standard BE test, comparing bioavailability, was not applicable to show the therapeutic equivalence between the spherical adsorbent carbon formulations acting inside the gastrointestinal tract without absorption. Setting clear clinical endpoints to evaluate their effects was not practical. The in vitro adsorption

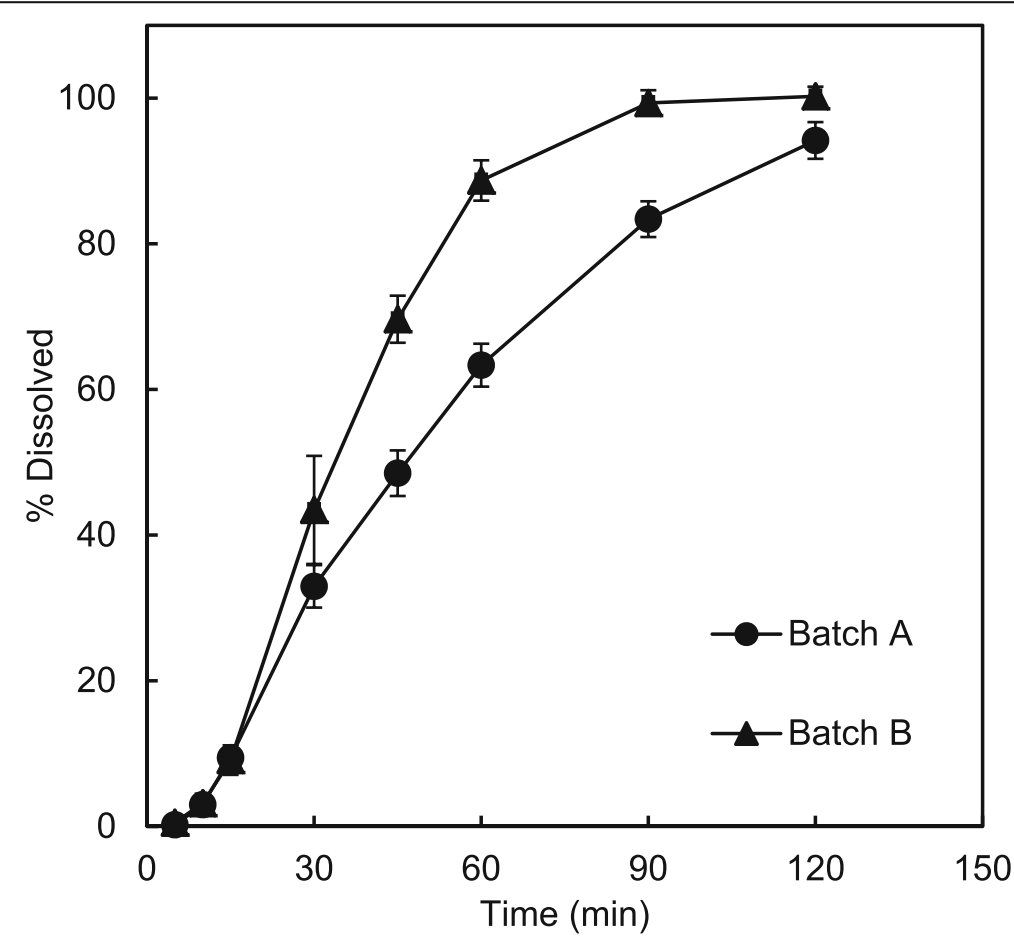

Fig. 1 Dissolution profiles of different batches of innovator itraconazole capsules in pH1.2 medium. Dissolution testing was conducted in an USP apparatus II using a volume of $900 \mathrm{~mL}$ of medium at an agitation rate of $50 \mathrm{rpm}$ 
Table 5 Pharmacokinetic parameters of itraconazole in plasma and $90 \%$ confidence interval of difference in the average values of logarithmic parameters

\begin{tabular}{llllll}
\hline Parameters & Number & $\begin{array}{l}\text { Reference product } \\
\text { (Batch A) }\end{array}$ & $\begin{array}{l}\text { Test product } \\
\text { (Batch B) }\end{array}$ & $\begin{array}{l}\text { Difference in the averate values } \\
\text { after logarithmic conversion }\end{array}$ & $\begin{array}{l}90 \text { \% Confidence interval of } \\
\text { difference in the average values } \\
\text { of logarithmic parameters }\end{array}$ \\
\hline $\mathrm{C}_{\max }(\mathrm{ng} / \mathrm{mL})$ & 28 & $41.5(23.6)$ & $64.2(23.4)$ & $\log (1.764)$ & $\log (1.415) \sim \log (2.198)$ \\
$\mathrm{AUC}_{48}(\mathrm{ng} \mathrm{h} / \mathrm{mL})$ & 28 & $318(166)$ & $453(161)$ & $\log (1.592)$ & $\log (1.317) \sim \log (1.926)$ \\
\hline
\end{tabular}

Data represents mean (SD). This test was performed by the innovator company

property of major uremic toxins (e.g., indole and indoleacetic acid) was also studied during the development of generic formulations without unified criteria.

Some publications, however, reported differences in physicochemical properties (e.g., in vitro absorption of indicator chemicals) and clinical effects between the originator and generic products (Miyazaki et al. 2008). A specific working group organized in the Committee, focusing on spherical adsorbent carbon found significantly lower adsorption of iodine and methylene blue to the generic products compared to the originator formulation (Table 6). The original product tended to show higher specific surface area, pore size, and pore volume, than the generic equivalent. Differences in adsorption against five major uremic toxins were also observed using in vitro adsorption isotherm measurements (Fig. 2), which was reported at the 4th meeting of the Committee (ECQGDP 2009). These data suggested potential differences in therapeutic effects between originator and generic products, while the clinical outcome study of a generic product conducted by the manufacturer indicated a certain degree of effectiveness. Thus, the manufacturers are trying to improve the formulations and reduce the variations in adsorption properties between formulations using appropriate indicator toxins, following the suggestion by the Committee.

\section{Other quality-related issues}

Other issues include some locally-acting drugs such as topical dermatological formulations (e.g., patches, ointments) and mesalazine (5-aminosalicylic acid) formulations (Braddy et al. 2015). Topical dermatological patches containing non-steroidal anti-inflammatory drugs (NSAID, e.g., diclofenac), available either as ethical medical formulations or over-the-counter products, are popular in Japan to relieve musculoskeletal pain, particularly in elderly patients. The current BE guideline include BE tests using the tape stripping and other in vitro methods to evaluate API release. Japanese Pharmacopoeia 17th Edition included adhesion tests. Many patients, however, identified differences in characters (performance) in the ethical generic formulations, which make appropriate selection of the generic products more difficult. It is often difficult to clearly distinguish the characteristics of pharmaceutically non-critical factors (e.g., simple preference of individual patients) and factors affecting the performance (API release). Information on the product characters should help choice of the products by pharmacist and patients.

\section{Future prospects}

\section{Characterization and BE studies of NBCD-generic/CGD}

\section{formulations}

Development of rational methods to confirm therapeutic equivalence of non-oral products and to evaluate a consistent formulation performance throughout the product lifetime are growing regulatory challenges. Many generic drug formulations containing complex APIs or non-oral route of administration that are not fully covered by current $\mathrm{BE}$ guidelines, should be categorized in Non-Biological Complex Drugs (NBCDs) or Complex Generic Drugs (CGDs), at least in their wider conception (Crommelin et al. 2015). Some of these formulations are relatively old or locally marketed products (e.g., iron-carbohydrate drugs and glatiramoids in the EU, spherical adsorbent carbon in Japan), while others use new technologies (e.g., liposomal drugs and nanomedicines).

Extensive physicochemical characterization by multiple methods, as reported for the low molecular weight

Table 6 Properties of spherical adsorbent carbon formulations

\begin{tabular}{|c|c|c|c|c|c|c|c|c|}
\hline & \multicolumn{2}{|c|}{$\begin{array}{l}\text { Adsorption property } \\
(\mathrm{mg} / \mathrm{g})\end{array}$} & \multicolumn{2}{|c|}{$\begin{array}{l}\text { Adsorption property after } \\
\text { crushing (mg/g) }\end{array}$} & \multirow[t]{2}{*}{$\begin{array}{l}\text { Specific surface } \\
\text { area }\left(\mathrm{m}^{2} / \mathrm{g}\right)\end{array}$} & \multirow[t]{2}{*}{$\begin{array}{l}\text { Micropore } \\
\text { diameter (A) }\end{array}$} & \multirow[t]{2}{*}{$\begin{array}{l}\text { Micropore volume } \\
\left(\mathrm{cm}^{3} / \mathrm{g}\right)\end{array}$} & \multirow[t]{2}{*}{ D50 (mm) } \\
\hline & lodine & Methylene blue & lodine & Methylene blue & & & & \\
\hline Originator & 1410 & 260 & 1400 & 310 & 1590 & 19.4 & 0.77 & 367.6 \\
\hline Generic 1 & 1280 & 50 & 1260 & 260 & 1260 & 16.5 & 0.52 & 302.0 \\
\hline Generic 2 & 1310 & 100 & 1320 & 260 & 1300 & 16.5 & 0.54 & 326.8 \\
\hline Medicinal Carbon & 1030 & 210 & 1070 & 210 & 1080 & 24.9 & 0.68 & 52.5 \\
\hline
\end{tabular}

The test was performed by JIS test methods for activated carbon 


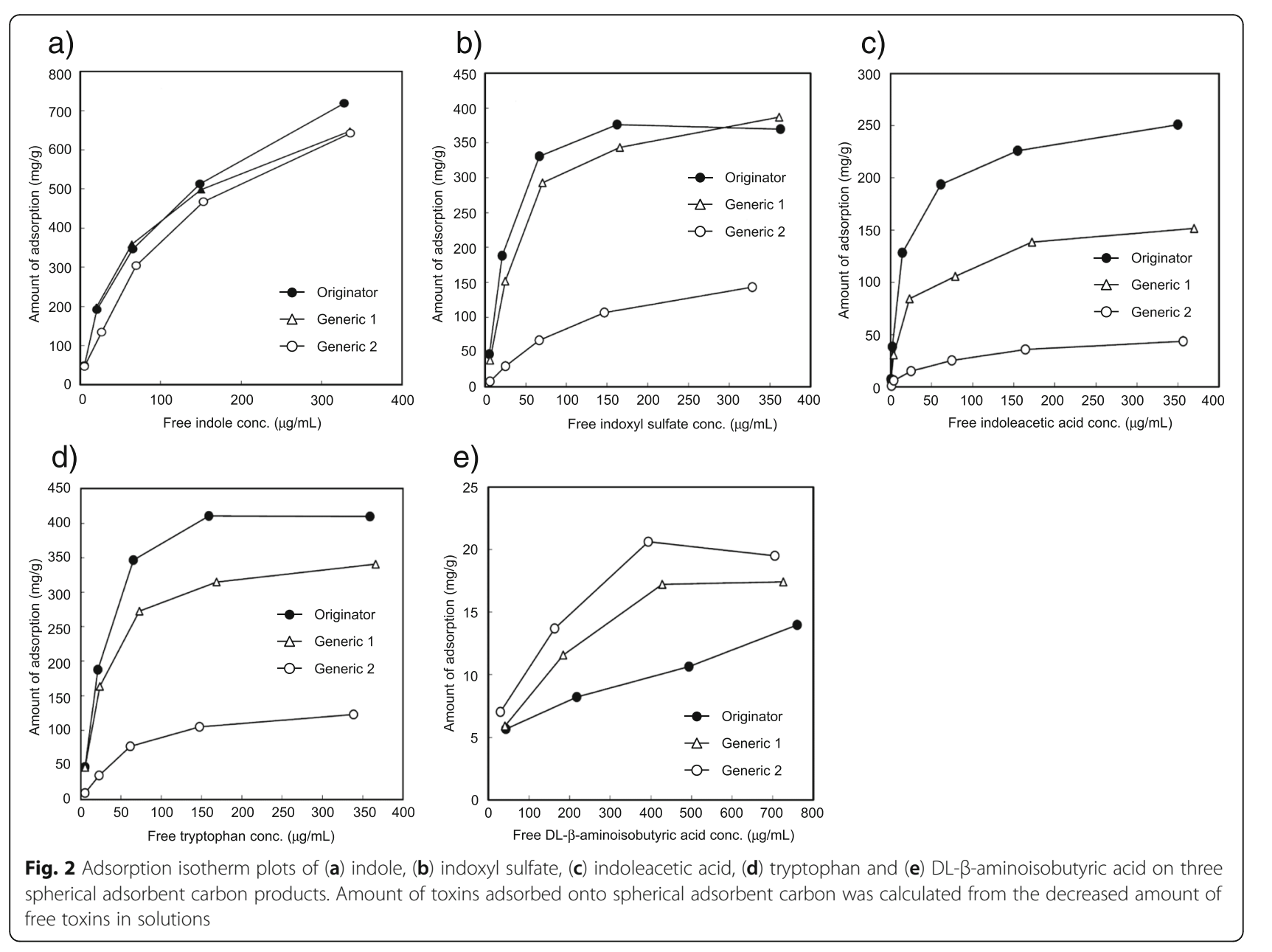

heparin, should be a promising way to ensure consistent quality and therapeutic equivalence of the CGD or NBCD generics (Crommelin et al. 2015; Schellekens et al. 2014; Ye et al. 2013). The improved characterization methods, particularly those showing critical elements influencing the therapeutic effects, should support both the product equivalence and the variation reduction between batches. in vitro methods to evaluate the performance of controlled release formulations, delivery systems using carriers, formulations administrated through non-oral routes, and drug-device combinations (e.g., inhalation and nasal spray) are becoming increasingly important for the development of new generic drug products (Chen et al. 2010). For example, a generic version of microsphere formulations containing leuprolide acetate was approved in 2014 in Japan. Providing BE guidelines, concept papers, or official testing methods in pharmacopeia would assist rational development of these formulations (MHLW 2016a; MHLW 2016b). Preparation of formulation-specific guidance indicating the required tests is under consideration in Japan, as a method to achieve BE between products and their quality in a rational development process. The concept of comparability exercise may be applied for the evaluation of certain nanomedicines such as liposome or block copolymer micelle products (Crommelin et al. 2015; Ehmann et al. 2013). Continuous provision of guidelines and recent improvement of the reviewing process at the new Office of Generic Drugs in PMDA should reduce the bioequivalence- and quality-related problems particularly for the complex generic drugs (Kuribayashi et al. 2016; MHLW 2016c). Since many new generic formulations are developed practically at the same time in different countries, sharing information between the regulatory bodies should be a viable way to reduce the required resources. Regulatory science studies, including development of new bioequivalence methods (e.g., GDUFA-funded research program in US), should support the product developments and bioequivalence (Yu et al. 2016).

\section{Conclusions}

Use of generic drugs is increasing continuously in Japan. Results obtained from the Formulation WG confirmed that the quality of generic products distributed in Japan are comparable to that of innovator products. We 
consider it very important to continue multiple approaches for the quality assurance of generic drugs, and to provide feedback the results to improve the measures for improving the reliability of generic drugs for both medical experts and patients.

\begin{abstract}
Abbreviations
ADME: Absorption, distribution, metabolism, elimination; API: Active pharmaceutical ingredient; AUC: Area under the curve;

BCS: Biopharmaceutics classification scheme; BE: Bioequivalence; CDER: Center for Drug Evaluation and Research; CGD: Complex generic drugs; $C_{\text {max }}$ : Peak plasma concentration; CTD: Common technical document; EP: European Pharmacopoeia; FIP: International Pharmaceutical Federation; FPMAJ: Federation of Pharmaceutical Manufacturers' Association of Japan; FY: Fiscal year; GMP: Good manufacturing practice; ICH: International Council for Harmonization; IM: Immediate release; ISO: International Organization for Standardization; JGA: Japan Generic Medicines Association; JP: Japanese Pharmacopoeia; MHLW: Ministry of Health, Labor, and Welfare; MR: Modified release; NBCD: Non-biological complex drugs; NIHS: National Institute of Health Sciences; NIID: National Institute of Infectious Diseases; NSAID: Non-steroidal anti-inflammatory drugs; OECD: Organization for Economic Cooperation and Development; OMCL: Official medicines control laboratories; PD: Pharmacodynamics; PIC/S: Pharmaceutical inspection convention and pharmaceutical inspection co- operation scheme; PK: Pharmacokinetic; PMDA: Pharmaceuticals and Medical Devices Agency; QRED: Quality reevaluation of ethical drugs; SUPAC: Scale-up and post-approval changes; WG: Working group
\end{abstract}

\section{Acknowledgements}

The authors thank the members and organizers of the Expert Committee on Quality of Generic Drug Products, and prefectural public health institutes of Aichi, Fukuoka, Hyogo, Kanagawa, Kyoto, Osaka, Saitama, Shizuoka, Toyama, and Tokyo, for collaboration and discussion.

\section{Funding}

Preparation of this manuscript was partly supported by the Research on Regulatory Harmonization and Evaluation of Pharmaceuticals, Medical Devices, Regenerative and Cellular Therapy Products, Gene Therapy Products, and Cosmetics Program from Japan Agency for Medical Research and Development (AMED).

\section{Authors' contributions}

The authors are former ( $\mathrm{CY}, \mathrm{HO})$ or current $(\mathrm{HS}, \mathrm{HY}, \mathrm{KI}, \mathrm{YG})$ members of Formulation Evaluation Working Group of the Expert Committee on Quality of Generic Drug Products. HS, HY and KI drafted the manuscript. CY is a current member of the parent Committee and provided information on the background of the program. YG and KI coordinated the study and manuscript revision. $\mathrm{HO}$ gave valuable comments on the draft and also polished it. All the authors read and approved the final manuscript.

\section{Competing interests}

The authors declare that they have no competing interests.

\section{Author details}

'Drug Division, National Institute of Health Sciences, 1-18-1 Kamiyoga, Setagaya-ku, Tokyo 158-8501, Japan. ${ }^{2}$ Osaka Office, Pharmaceutical and Medical Device Regulatory Science Society of Japan, 2-1-2 Hiranocho, Chuo-ku, Osaka 541-0046, Japan.

Received: 8 July 2016 Accepted: 26 September 2016

Published online: 07 October 2016

\section{References}

Baert B, De Spiegeleer B (2010) Quality analytics of Internet pharmaceuticals. Anal Bioanal Chem 398:125-136

Braddy AC, Davit BM, Stier EM, Conner DP (2015) Survey of international regulatory bioequivalence recommendations for approval of generic topical dermatological drug products. AAPS J 17:121-133
Chen ML et al (2010) Challenges and opportunities in establishing scientific and regulatory standards for assuring therapeutic equivalence of modified-release products: workshop summary report. Eur J Pharm Sci 40:148-153

Crommelin DJ et al (2015) The similarity question for biologicals and non-biological complex drugs. Eur J Pharm Sci 76:10-17

Davit B, Braddy AC, Conner DP, Yu LX (2013) International guidelines for bioequivalence of systemically available orally administered generic drug products: a survey of similarities and differences. AAPS J 15:974-990

ECQGDP (2009) Report on quality of spherical adsorbent carbon formulations. http://www.nihs.go.jp/drug/ecqaged/shiryou4-1-2.pdf. Accessed 25 Dec 2009

ECQGDP (2010) Report on bioequivalence study of innovator itraconazole formulation batches. http://www.nihs.go.jp/drug/ecqaged/shiryou5-2-3.pdf. Accessed 15 Sept 2009

ECQGDP (2016) Expert Committee on Quality of Generic Drug Products webpage. http://www.nihs.go.jp/drug/ecqaged.html

Ehmann F et al (2013) Next-generation nanomedicines and nanosimilars: EU regulators' initiatives relating to the development and evaluation of nanomedicines. Nanomedicine (Lond) 8:849-856

FIP Biowaiver monographs. http://www.fip.org/bcs_monographs

Fujimura S, Watanabe A (2012) Generic antibiotics in Japan. J Infect Chemother 18:421-427

García-Arieta A, Gordon J (2012) Bioequivalence requirements in the European Union: critical discussion. AAPS J 14:738-748

Janssens S, Van den Mooter G (2009) Review: physical chemistry of solid dispersions. J Pharm Pharmacol 61:1571-1586

Katori N (2014) Accession to the PIC/S and pharmaceutical quality system in Japan. (In Japanese). Kokuritsu lyakuhin Shokuhin Eisei Kenkyusho Hokoku 132:22-35

Khan MH, Tanimoto T, Nakanishi Y, Yoshida N, Tsuboi H, Kimura K (2012) Public health concerns for anti-obesity medicines imported for personal use through the internet: a cross-sectional study. BMJ Open 2:e000854

Kuribayashi R, Matsuhama M, Mikami K (2015) Regulation of generic drugs in Japan: the current situation and future prospects. AAPS J 17:1312-1316

Kuribayashi R, Takishita T, Mikami K (2016) Regulatory considerations of bioequivalence studies for oral solid dosage forms in Japan. J Pharm Sci 105:2270-2277

MHLW (2012a) Guideline for Bioequivalence Studies. http://www.nihs.go.jp/drug/ DrugDiv-J.html. Accessed 29 Feb 2012

MHLW (2012b) Promotion of the Use of Generic Drugs. http://www.mhlw.go.jp/ english/policy_report/2012/09/120921.html

MHLW (2016a) Concept paper for bioequivalence studies of generic aqueous ophthalmic solutions (In Japanese). https://www.pmda.go.jp/files/000210453. pdf. Accessed 11 Mar 2016

MHLW (2016b) Concept paper for bioequivalence studies of generic dry powder inhalers (In Japanese). https://www.pmda.go.jp/files/000210452.pdf. Accessed 11 Mar 2016

MHLW (2016c) Guideline for the development of liposome drug products. http://www.nihs.go.jp/drug/section4/160328_MHLW_liposome_guideline.pdf

Miyazaki Y, Yakou S, Asayama T, Kouno H, Sakurai S, Yanagawa C (2008) Comparison of physicochemical and adsorptive properties of brand-name and generic spherical carbon adsorbents (In Japanese). Jpn J Pharm Health Care Sci 34:1077-1085

Morihara M, Aoyagi N, Kaniwa N, Kojima S, Ogata H (2001) Assessment of gastric acidity of Japanese subjects over the last 15 years. Biol Pharm Bull 24:313-315

MUFJRC (2014) Study report on measures to improve reliability of generic drug products (In Japanese). http://www.mhlw.go.jp/bunya/iryou/kouhatu-iyaku/ dl/h24-02_1.pdf

OECD (2010) Generic Pharmaceuticals 2009. https://www.oecd.org/competition/ abuse/46138891.pdf. Accessed 5 Oct 2010

OECD (2015) Data on elderly population. https://data.oecd.org/pop/elderlypopulation.htm

PMDA (2014) Guidance for generic drug approval process (In Japanese). http://www.pmda.go.jp/files/000161866.pdf

Schellekens $\mathrm{H}$ et al (2014) How to regulate nonbiological complex drugs (NBCD) and their follow-on versions: points to consider. AAPS J 16:15-21

Takizawa O, Urushihara H, Tanaka S, Kawakami K (2015) Price difference as a predictor of the selection between brand name and generic statins in Japan. Health Policy 119:612-619

Toyoguchi S et al (2005) Comparizon of itraconazole formulations (2). J New Rem Clin 54:1408-1412

Ueda H, Shibahara N, Takagi S, Inoue T, Katsuoka Y (2007) AST-120, an oral adsorbent, delays the initiation of dialysis in patients with chronic kidney diseases. Ther Apher Dial 11:189-195 
Umezawa S, Fujita H (2013) Generic intravenous ritodrine hydrochloride (Rindolf ${ }^{\mathrm{T}}{ }^{\mathrm{M}}$ ) causes liver injury more frequently than original ritodrine hydrochloride (Utemerin ${ }^{\top M}$ ) (In Japanese). J Jpn Soc Perin Neon Med 49:288-294 Uppoor RS, Vaidyanathan J, Mehta M, Yu LX (2014) Biowaiver and biopharmaceutics classification system. In: Yu LX, Li B (eds) FDA Bioequivalence Standards, vol 13. Springer, New York, pp 119-137

Vial J, Cohen M, Sassiat P, Thiébaut D (2008) Pharmaceutical quality of docetaxel generics versus originator drug product: a comparative analysis. Curr Med Res Opin 24:2019-2033

WHO (2011) General notes on Biopharmaceutics Classification System (BCS)-based biowaiver applications., http://apps.who.int/prequal/info_applicants/BE/BW_ general_2011November.pdf

Ye H et al (2013) Characterization of currently marketed heparin products: key tests for LMWH quality assurance. J Pharm Biomed Anal 85:99-107

Yomota C (2012) Trends in the quality evaluation of generic products and bioequivalence guidelines (In Japanese). Kokuritsu lyakuhin Shokuhin Eisei Kenkyusho Hokoku 130:1-12

Yu L et al (2016) Advancing product quality: a summary of the second FDA/PQRI conference. AAPS J 18:528-543

\section{Submit your manuscript to a SpringerOpen ${ }^{\circ}$ journal and benefit from:}

- Convenient online submission

- Rigorous peer review

- Immediate publication on acceptance

- Open access: articles freely available online

- High visibility within the field

- Retaining the copyright to your article

Submit your next manuscript at $\gg$ springeropen.com 